\title{
Problem Posing and Its Relationship with Teaching Experience of Elementary School Mathematics Teachers from Ethnic Minority Area in Southwest China
}

\author{
Aihui Peng ${ }^{1 *}$ (D), Mengdie $\mathrm{Li}^{1}$ (D), $\operatorname{Lin}_{\operatorname{Lin}}{ }^{1}$ (D), $\mathrm{Li} \mathrm{Cao}^{2}$ (D), Jinfa $\mathrm{Cai}^{3}$ (D) \\ 1 Southwest University, CHINA \\ 2 University of West Georgia, USA \\ 3 University of Delaware, USA
}

Received 27 July 2021 - Accepted 16 November 2021

\begin{abstract}
This study examined characteristics of problem posing and its relationship with the teaching experience of elementary school mathematics teachers from ethnic minority areas in the Southwest of China. Eighty-one $(N=81)$ elementary school mathematics teachers responded to a task-based questionnaire and posed mathematical problems based on given problem situations. Characteristics of their mathematical problem posing were observed through the total numbers, appropriateness, difficultly, and flexibility levels of the problems posed by the teachers. Results show that elementary school mathematics teachers were capable of posing a considerable number of appropriate mathematical problems. Significant differences were found in the flexibility of the posed problems among the teachers with different years of teaching experience. This finding suggests that teachers' capability in mathematical problem posing may not necessarily grow along with teaching experience. Limitations of the study and implications in developing teachers' mathematical problem posing are discussed.
\end{abstract}

Keywords: mathematical problem posing, elementary school, mathematics teacher development, teaching experience, ethnic minority, mathematical representation

\section{INTRODUCTION}

Problem posing has long been recognized as a critically important intellectual activity in a scientific investigation. Eminent mathematicians and scientists underscored the importance of problem posing in advancing and deepening knowledge and understanding. As Einstein and Infeld (1938) posited, the "formulation of a problem is often more essential than its solution, which may be merely a matter of mathematical or experimental skill. To raise new questions, new possibilities, to regard old problems from a new angle, require creative imagination and marks real advance in science" (p. 95). In mathematics, the art of posing a question be held as high or higher in value than solving it; and similarly, in the history of science, formulating precise, answerable questions not only advances new discoveries but also gives scientists intellectual excitement (Cai \& Mamlok-Naaman, 2020). In the past decades, an increasing number of mathematics educators and researchers have also argued for the importance of getting students to pose problems. Some countries have included problem posing in the mathematical curricula standards which serve as the leading document to shape future of the mathematics education in these countries (Li et al., 2020). As problems provide students with opportunities to do and learn mathematics and they convey messages about the nature of the discipline - what it entails and what is worth knowing and doing (Schoenfeld, 1989), it is widely agreed that mathematics teachers be able to effectively pose problems to develop students' problem posing ability. Good problem posing involves constructing problems that are meaningful for students and also addressing the underlying mathematical principles (Brown, 2001). Problem posing, therefore, serves as a productive field to exercise teaching competence (Crespo, 2003). Cai and Hwang (2021) strongly argued that teachers can play a leading role in co-creating the innovative curriculum materials as re-

(c) 2022 by the authors; licensee Modestum. This article is an open access article distributed under the terms and conditions of the Creative Commons Attribution License (http://creativecommons.org/licenses/by/4.0/). 


\section{Contribution to the literature}

- This study contributed to our understanding of the characteristics of problem posing of an underrepresented group, elementary school mathematics teachers from ethnic minority areas in the Southwest of China.

- The study added new knowledge on the cultural features of the problem posed by elementary school mathematics teachers with an ethnic minority background.

- The findings show that teaching experience was not necessarily human capital in mathematical problem posing for the group, which contradicted the traditional view of the value of teaching experience in educational practice.

designers of curriculum to teach mathematics through problem posing. Similarly, Liljedahl and Cai (2021) suggested that future direction should be put on problem posing as an opportunity to teach mathematics.

Despite the calls for the importance of mathematics problem posing, recent research revealed that there is a lack of experience in mathematics problem posing among prospective teachers (Leavy \& Hourigan, 2021). Their experience was often limited to traditional problem solving, i.e., closed problems focusing on arithmetic operations (Leavy \& Hourigan, 2020). Accordingly, prospective and practicing teachers may have little experience or insights into what constitutes a mathematically worthwhile problem. As these studies clearly show, experience impacts mathematics teachers' problem posing. This shortcoming of mathematics teacher training and development can be traced back to earlier studies that highlighted teaching experience as a significant influence on teaching competency and student learning. For instance, in a multiple-regression study to investigate factors of educational achievement, Schmitt (2013) found that teachers with over 30 years of experience had a negative association with students' achievement in math. This result was interpreted as the inability of the teachers to keep up with instructional skills in education. Similarly, using a Likert-type survey and a cross-sectional design, Xie and Cai (2020) explored the relationship between high school mathematics teachers' beliefs and their teaching experience. They found that teachers with 6 to 10 years of experience exhibit the most desirable beliefs as compared with other groups of teachers. These results demonstrate the association of teaching experience with teaching competence and student learning outcome. However, it remains unclear whether such a relationship applies to teachers' mathematical problem posing and in which way (if there is). This study focused on the association of the years of teaching experience with mathematics teachers' problem posing.

Furthermore, it is widely recognized that mathematics is a cultural product, particularly in mathematics education. This view has generated a wealth of data from the anthropological and crosscultural perspectives and led to a growing consensus for diversity in mathematics education in recent years
(Nasir et al., 2008; Venkat \& Askew, 2018). Acceptance and understanding of cultural diversity in developing education policy is not optional, but necessary (Ogbu, 1995). China operates the world's largest education system, including educating students from China's 55 official ethnic minority groups. Research on mathematics teachers' problem posing was mainly conducted by investigating teachers from the dominant culture and very little on teachers from underrepresented cultures (Peng, 2021). As Graham (1988) stated, equitable mathematics education is not simply about replicating the equality of opportunities and equity of outcomes based on the dominant, hegemonic group. As mathematical problem posing is an important indicator of teacher competency and an important determinant of students' mathematics learning, it is important to understand the characteristics of the mathematics teachers from the ethnic minorities in China. Studying problem posing of mathematics teachers from the minority group would enrich the research on mathematics problem posing and mathematics education from a cultural perspective. The current study addressed this research gap by investigating the mathematical problem posing of the elementary school mathematics teachers from ethnic minority areas in the Southwest of China.

Specifically, the current study addressed two questions: (1) What are the characteristics of problem posing of elementary mathematics teachers from ethnic minority areas in the Southwest of China? (2) What is the relationship between problem-posing and the teaching experience of these teachers?

\section{THEORETICAL PERSPECTIVES}

\section{Mathematics Problem Posing}

With the recent rapid development in research on mathematics problem posing, the connotations for problem posing also developed. Liljedahl and Cai (2021) presented three perspectives on problem posing: as a cognitive activity, a learning goal, and an instructional approach. This conceptual development continued to nuance the state-of-the-art research in mathematics education. Baumanns and Rott (2020) developed a framework for the characterization of problem-posing 
activities that linked three theoretical constructs from research on problem posing, problem-solving, and psychology: (1) problem-posing as an activity of generating new or reformulating given problems, (2) emerging tasks on the spectrum between routine and non-routine problems, and (3) metacognitive behaviour in problem-posing processes.

Among the different connotations above, that core view that problem posing is important for nurturing students' mathematical thinking remains the same. Historically, Silver's definition was the most widely accepted one, which is defined as a cognitive activity of either re-formulating given problems or generating new problems (Silver, 1994). Under this definition, problem posing can occur before, during, or after the solution of a problem. Problem re-formulation usually occurs within the process of problem solving when the problem solver engages in recreating a given problem in some ways to make it more accessible for a solution. Generating new problems occur at times when the goal is not the solution of a given problem, but the creation of a new problem from a situation or experience. Such problem posing can occur before any problem solving. This definition highlights the problem posing as "a feature of creative activity or exceptional mathematical ability" and is widely accepted in the research community. However, as the research of problem posing accumulates, it is necessary to modify the definition to include different manifestations of problem posing (Cai \& Hwang, 2020). Along this line, Cai and Hwang (2020) posited:

\section{"By problem posing in mathematics education, we refer to several related types of activity that entail or support teachers and students formulating (or reformulating) and expressing a problem or task based on a particular context (which we refer to as the problem context or problem situation)" (p. 2).}

In this definition, problem posing becomes more inclusive. It represents multiple dimensions of mathematical problem posing, describes the role of teachers and students in the problem posing process, and highlights the importance of the context for problem posing. Furthermore, Cai and Hwang (2020) emphasized that problem and task are broadly defined to include any mathematical question that can be asked and any mathematical task that can be performed based on the problem situation. They stressed that context, also broadly defined, includes within-mathematics situations, as well as situations drawn from external referents such as real-life phenomena and questions from other disciplines. Baumanns and Rott (2021) further gave a comprehensive account of the different situations used for problem posing. By reviewing 271 potential situations for problem posing from 241 systematically gathered articles on problem posing, they characterized the situations from the following three aspects: (1) levels of openness of the situation; (2) situations are assigned to categories free, semi-structured, and structured; (3) for the structured situations, they are further categorized according to whether they are routine or non-routine problems. These different characteristics provide insights into the disparities of the different situations for problem posing. The current study adopts these two theoretical frameworks. First, we adopted Cai and Hwang's (2020) definition of problem posing. This conceptual description allowed us to see problem posing as specific intellectual activities of a particular group. In our case, teachers themselves pose mathematical problems based on given problem situations which may include mathematical expressions or diagrams (Cai \& Hwang, 2020). Second, we used Baumanns and Rott's (2021) characterization to examine and measure the situations for problem posing in our study.

\section{Measures of Mathematical Problem Posing}

Multiple indicators have been used to measure problem posing. In earlier work, Balka (1974) analyzed responses of students' mathematical problem posing according to fluency, flexibility, and originality. Fluency refers to the number of problems posed or questions generated. Flexibility denotes the number of different categories of problems generated. Originality indicates how rare the response is among all responses. These categories are commonly used in the studies when students' creativity is examined with problem posing (e.g., Van Harpen \& Sriraman, 2013).

Silver and Cai (2005) suggested similar criteria but used slightly different terminologies that are more mathematical context-based: quantity, originality, and complexity. Quantity refers to the number of solvable mathematical problems, similar to fluency in Balks (1974). The originality of the problem posed by students can be measured by its rareness, similar to the originality in Balka (1974). Complexity reveals the depth of mathematical thinking in problem posing and has been considered by many researchers as a crucial criterion (Guo et al., 2020). Silver and Cai (1996) operationalized the mathematical complexity through the presence of assignment, relational, and conditional propositions in the problem statements. An assignment proposition is a question such as "How many miles did they drive in all?" A relational proposition is a statement such as "How many more miles did Arturo drive than Jerome?" A conditional proposition is a question such as "If Arturo drove 80 miles more than Elliot, how many miles did Arturo drive?" Problems with the conditional and relational propositions tend to be more difficult for students to solve than those containing only the assignment propositions. Thus, the presence of conditional or relational propositions can serve as an indication of problem complexity. In Silver and Cai's (1996) framework, complexity is measured through the 
difficulty level of the problem posed. But, it does not contain the category of flexibility as compared with Balka' (1974). According to Li et al. (2020), the number of different difficulty levels of the problems posed can be used as an indicator of the variety of the problems posed. This number therefore can serve as an indicator of the flexibility in problem posing.

In addition to the above, there are a few less commonly-used indicators for measuring mathematical problem-posing such as correctness, appropriateness, and accuracy. For instance, Nicolaou and Xistouri (2011) used correctness to evaluate students' problem-posing, a criterion similar to quantity in Silver and Cai's (2005) study. De Corte and Verschaffel's (1996) defined a problem as appropriate if it contains a question, requires a prescribed computation, is answerable, includes the given numbers, is concrete (e.g., the problem "Mother has to divide 0.6 by 0.8 . Can you help her?" was evaluated as not concrete), and uses the numbers correctly (e.g., " 4.8 students" was an example of the incorrect use of the number 4.8). Regarding accuracy, Guo et al. (2020) defined it as the proportion of solvable mathematical problems posed. Among these indicators, quantity and complexity are the most frequently used criteria (Cai \& Hwang, 2002; Guo et al., 2020; Peng et al., 2020).

In the current study, both situation-given and mathematical expression-given tasks were provided for teachers to pose mathematical problems. We followed Silver and Cai's (1996) conception of complexity to observe the difficulty and the flexibility level of the problem posed by teachers. We also examined the appropriateness of the posed problems by calculating the total numbers of posed problems minus both the incorrect and repeated problems.

\section{Research on Mathematics Teachers' Problem Posing}

Recognized as an important attribute of teaching competence, considerable studies have been conducted on mathematics teachers' problem posing. These studies typically provide a problem situation and ask teachers to pose problems that can be solved using the information given in the situation. Such studies have explored the performance of both the preservice and in-service teachers and generated two lines of research. On one line, many studies supported the claim that both the preservice and in-service teachers are capable of posing interesting and important mathematical problems (Cai \& Hwang, 2020). For instance, Li et al. (2019) examined 83 Chinese elementary school mathematics teachers of the total number of problems posed, percent of appropriate problems, difficulty levels, and flexibility levels. They found that most teachers are capable of posing interesting problems. Similarly, more intervention studies reported that problem-posing workshop has great potential in enhancing teachers' problem posing
(Cai et al., 2019; Li et al., 2019, 2020). A few more studies in a recent special issue edited by Cai and Hwang (2020) also support this growing consensus. Together, this line of research leveraged multiple perspectives on problem posing and elaborated on its importance in a pedagogical context.

On the other line, studies were focused on a specific area of mathematics education, predominantly in Fraction. Results from these studies show that a deficiency of knowledge of problem posing limits teachers' performance in problem posing. For instance, De Corte and Verschaffel (1996) administered a paperand-pencil test to 214 school children and 99 pre-service mathematics teachers and asked them to pose word problems whose solutions involved six multiplication and six division number sentences. They found that the teachers did as poorly as the students when posing problems for sentences. Toluk-Usar (2009) also reported a study that used arithmetical problems posing as an intervention to enhance understanding of Fractions among 95 Turkish pre-service mathematics teachers. His results show many participants found it difficult to pose problems involving any arithmetic operation with fractions. Similarly, Tichá and Hošpesová (2013) asked pre-service teachers to pose problems containing some given data (namely fractions). Analyses of the problems posed by the pre-service teachers revealed shortcomings in their conceptual understanding of fractions. Together, the results of these studies point to problem posing as a problematic area of professional development for mathematics teachers.

\section{Mathematics Teachers' Problem Posing and Teaching Experiences}

As indicated earlier, research studies on problem posing have increased in recent years, but its relationship with teaching experience remains unclear. To our knowledge, Klein and Leikin (2020) is the only study that considered this relationship. However, this study focused on the link between teachers' conceptions and open mathematical problems in teaching and learning. Their results show that teaching experience had a significant effect on teachers' conceptions related to the difficulty of posing open tasks: Teachers with 1 to 4 years of teaching experience considered posing open tasks more difficult than those with over 9 years of teaching experience. Nevertheless, regarding the relationship between posed problems and teaching experiences, they did not conduct inferential statistics and concluded: "that years of experience had less influence on the type of posed open tasks since the distributions were similar" (p. 358). This result suggests that more research is needed to clarify the relationship between teachers' problem posing and teaching experience. A recent special issue edited by Cai and Leikin (2020) reported an increasing research interest in exploring the effect in mathematics teachers' 
mathematical problem posing and ways to develop the competency of problem posing among mathematics teachers. The current study joined forces with this research by exploring factors that influence teachers' competence in mathematical problem posing.

In response to the increased research interest in mathematical problem posing and the calls for developing teachers' problem posing ability (Cai \& Hwang, 2020; Cai \& Leikin, 2020), research has already started to examine mathematics teachers' problem posing (Lee, 2020). However, the existing research is largely focused on problem posing among the preservice and in-service teachers from the mainstream groups. Few studies investigated the problem posing of the teachers from the under-represented groups (Lee, 2020). To our knowledge, this is the only study that examined teaching experience, as an indicator of a teacher's human capital, in mathematical problem posing of the elementary school mathematics teachers from ethnic minority areas in the Southwest of China.

\section{RESEARCH DESIGN AND METHODS}

This study was part of a larger project to investigate educational quality in ethnic minority areas in the Southwest of China. One of the purposes of this subproject was to examine mathematics teachers' professional competency, including mathematical problem posing, and to inform appropriate development of teacher training programs for this teacher group.

\section{Participants and Data Collection}

The participants included 81 mathematics teachers from five elementary schools in two counties in a Southwest province of China. They participated voluntarily in the study during a professional development program provided by teacher educators from the local educational bureau in the winter of 2018. Before the administration of the questionnaire, the instructions were read aloud to them. They were informed of the purpose of the study and ensured that all information they provided will be kept confidential. Any personally identifiable information in the data will be removed and only the non-traceable results will be shared in a summary form. In addition to the instruction, the questionnaire included three parts: instruction, demographic variables, and tasks for problem posing. The instruction section briefly described the purpose of the study and the related ethical issues. The demographic variables included participants' gender, years of teaching experiences, degree, their ethnicities, and the schools where they were located. The tasks section presented four tasks for problem posing. More detailed descriptions of these tasks are presented in the next section. Before presenting the tasks, the participants were encouraged to pose as many mathematical problems as they can. It took about thirty minutes to
Table 1. The demographic information of the participants

\begin{tabular}{llcc}
\hline & & Number & Percent \\
\hline Gender & Male & 25 & $30.5 \%$ \\
Years of teaching & Female & 56 & $69.5 \%$ \\
experience & $1-5$ & 8 & $9.9 \%$ \\
& $6-15$ & 34 & $42 \%$ \\
& $16-25$ & 25 & $30.9 \%$ \\
Degree & Above 25 & 14 & $17.3 \%$ \\
& Junior college & 28 & $34.1 \%$ \\
& Bachelor & 53 & $65.9 \%$ \\
\hline
\end{tabular}

complete the questionnaire during which the teacher educators were present. Table 1 presents demographic information of the participants who were divided into four groups based on teaching experience of i.e., 1-5 years $(n=8), 6-15$ years $(n=34), 16-25$ years $(n=25)$, and $25+$ years $(n=14)$.

\section{The Tasks in the Questionnaire}

There were four tasks in the questionnaire, which included two situation-given tasks (Task 1 and Task 3) and two mathematical expression-given tasks (Task 2 and Task 4). According to Baumanns and Rott (2021), Task 1 and Task 3 can be identified as free situations, whereas task 2 and task 4 can be identified as semistructured situations. These tasks were adapted from published studies (Cai \& Hwang, 2002; Li et al., 2019; Li et al., 2020). They were chosen for the current study because they were widely used in classic literature on problem posing and had been specifically used both for students and elementary teachers in China. Specifically, Task 1 was adapted from Cai et al. (2020) and focused on distance and time on driving. This driving task was used for teachers' predictions of their students' problem posing. Results of their study showed that: teachers posed 390 problems in total; $50 \%$ of the teachers posed more than eight problems; $99 \%$ of the proposed problems were viable mathematical problems; only seven of them were too vague as to be incomprehensive. Task 3 was adapted from Cai and Hwang (2002). Their results showed that students in the two samples tended to move away from generating problems solely about the given information as they generated problems of greater difficulty. Both task 2 and task 4 were adapted from $\mathrm{Li}$ et al. (2019), in which the results showed that teachers posed 168 problems and 161 problems with various levels of difficulties, respectively. Figure 1 shows the information on these tasks.

\section{Data Analysis Procedures}

\section{Coding methods}

The coding of this study was adapted and built on the coding schemes of the previous studies (Li et al., 2019; Silver \& Cai, 1996). First, we determined whether each problem posed by the teachers was a valid problem. A posed problem was deemed valid if it was a 
Task1: Driving Task

Xiaobin, Shasha, and Jiajia are driving home from a trip. Jiajia drove 150 miles less than Shasha. Shasha drove twelve times as far as Xiaobin drove. Xiaobin drove 50 miles.

Task 2: $\quad 1 \frac{3}{4} \div \frac{1}{2}$

Task 3: Doorbell task

Sally is having a party. The first time the doorbell rings, 1 guest enters. The second time the doorbell rings, 3 guests enter. The third time the doorbell rings, 5 guests enter. The fourth time the doorbell rings, 7 guests enter. Keep on going in the same way. On the next ring a group enters that has 2 more persons than the group that entered on the previous ring.

Task 4: $6 \mathrm{x}+5=25$

Figure 1. The problem-posing tasks

Table 2. Coding examples for the posed problems in Task 1 (driving task) by a teacher

\begin{tabular}{|c|c|}
\hline Examples of the problem posed in task 1 & Difficulty levels \\
\hline How many miles did Shasha drive? & 1 (assignment) \\
\hline How many miles did Jiajia drive? & 1 (assignment) \\
\hline What is the ratio of the miles of Shasha's driving and Xiaobing's driving? & 2 (relational) \\
\hline How many miles in total for them to drive home? & 2 (relational) \\
\hline $\begin{array}{l}\text { If the average speed is } 100 \text { miles per hour, how many hours do they drive, and how long it will take to go } \\
\text { home? }\end{array}$ & 3 (conditional) \\
\hline If they drive 8 hours a day, can they go home within one day? & 3 (conditional) \\
\hline $\begin{array}{l}\text { If they get off at } 7 \text { o' clock in the morning and rest for } 2 \text { hours after driving for a period, when can they } \\
\text { arrive home? }\end{array}$ & 3 (conditional) \\
\hline
\end{tabular}

mathematical problem that was related to the task and its wording was comprehensible. A problem was considered invalid if it was nonmathematical, incomprehensibly worded, unrelated to the task, or simply a statement. Second, we coded and tallied the total number of the posed problems and the appropriate problems, the difficulty and flexibility levels of the posed problems.

We used Silver and Cai's (1996) coding for the mathematical complexity of each posed problem. We focused on the presence of assignment, relational, and conditional propositions in the problem statements. We further coded the difficultly levels as 1 (Low), 2 (Medium), and 3 (High), according to the presence of assignment, relational, and conditional propositions in the posed problem. Finally, we coded the flexibility level of the posed problem as level 1 (Low), 2 (Medium), and 3 (High), when there was only one difficulty level in a posed problem, two levels of difficulty levels, and three levels of difficulty levels, respectively. Table 2 provides a coding example for the problem posed in Task 1 (driving task) by a teacher. As can be seen, the teacher posed seven problems in total. Of which, there are two problems with an assignment proposition, two problems with a relational proposition, and three problems with a conditional proposition. Therefore, these posed problems were coded as two problems at low (1) difficulty level, two at medium (2) difficulty level, and three at high (3) difficulty level. For this task, the difficulty level and flexibility level were both coded at the high (3) level.

Following the schemes in Table 2, a systemic codebook was developed. Two graduate research assistants coded the data independently. They worked collaboratively to resolve disagreements on data and coding through discussions, developed a common codebook to process the data, and generated initial findings. The first author supervised the coding procedure and double-checked all the codes to ensure accuracy and consistency. All the data were kept in a spreadsheet file and then transferred into SPSS.

\section{RESULTS}

The data were analyzed using SPSS (Version 24.0). After the check for outliers, normality, homogeneity, and missing values, descriptive statistics were used to depict characteristics of problem posing of the elementary school mathematics teachers from ethnic minority areas. Then, one-way ANOVA was conducted to examine the relationship between mathematics teachers' problem posing and their teaching experience. 
Table 3. The total numbers, the appropriate problems, difficulty levels, flexibility levels of problems posed by mathematics teachers

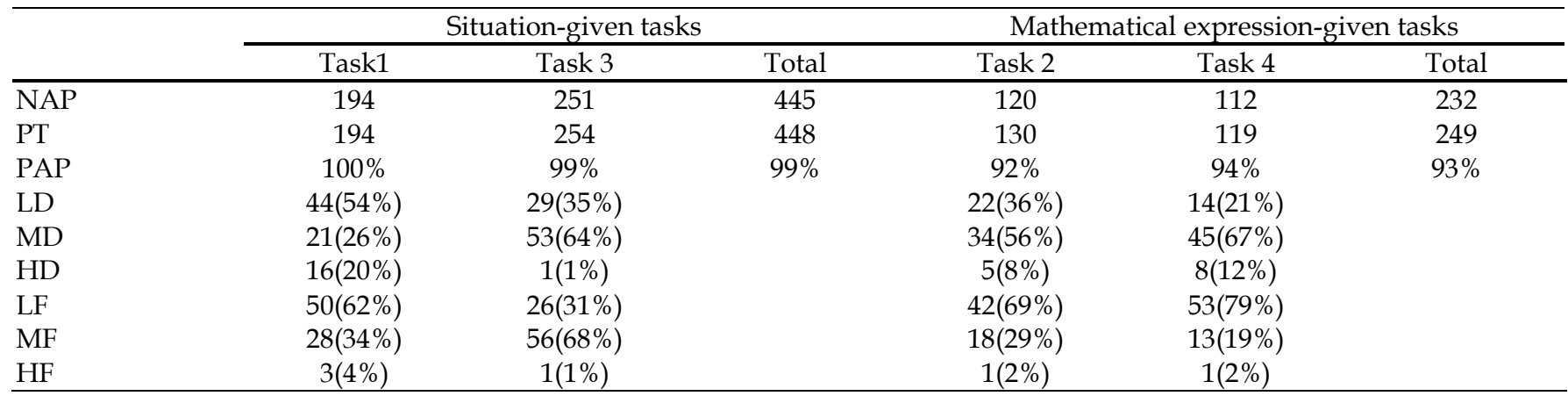

Note. NAP $=$ Numbers of Appropriate Problems Posed. PT $=$ Problems Posed in Total. PAP $=$ Percent of Appropriate Problems. LD = Low (difficulty) level of Posed Problems. MD = Medium (difficulty) level of Posed Problems. HD = High (difficulty) level of Posed Problems. LF= Low (flexibility) level of Posed Problems. MF = Medium (flexibility) level of Posed Problems. HF = High (flexibility) level of Posed Problems

\section{Characteristics of Mathematics Teachers' Problem Posing}

The total numbers, difficulty levels, and flexibility levels for each posed problem, for each teacher, and different types of tasks were calculated and presented in the tabular form to depict characteristics of the mathematics teachers' problem posing. The results are presented below.

\section{The total numbers and the appropriate problems posed by mathematics teachers}

Table 3 presents the distribution of the total numbers and the appropriate problems posed by the mathematics teachers. As Table 3 shows, participants posed a total of 448 problems for the situation-given tasks and 249 problems for the mathematical expression-given tasks. There are high percentages of appropriate problems for both situation-given tasks (99\%) and mathematical expression-given tasks (93\%). Our data further show that the participants performed differently in the two different types of tasks. In the two situation-given tasks (\#1 and \#3), they posed one to seven problems. For Task 1 (Driving task), many teachers posed up to five problems that are both correct and non-repetitive. For Task 3 (Doorbell task), all teachers posed at least one problem, and some posed seven problems. Whereas for the mathematical expression-given tasks (\#2 and \#4), the participants were able to pose up to five problems in various situations such as shopping, engineering, etc. However, they posed more repeated and incorrect problems for both Task 2 (Fraction) and Task 4 (Equation). In general, they performed better on the situation-given tasks than on the mathematical expression-given tasks. This result suggests it was easier for them to pose problems for the situation-given tasks than for the mathematical expression-given tasks $(t=$ 5.094, $p<0.001$, Cohen's D = 0.864).

\section{The difficulty levels of problems posed by mathematics teachers}

Table 3 presents the difficulty levels of problems posed by mathematics teachers. As can be seen in Table 3 , the difficulty levels of the problem posed are mostly at low and medium levels, and there are fewer problems at the high difficulty level. Also, the difficulty level of the problem posed varied in the situation-given tasks and the mathematical expression-given tasks. Specifically, there is a higher percentage of problems with a low difficulty level in situation-given tasks than in the mathematical expression-given tasks. This result suggests that teachers intended to pose problems with high-level difficulty in the mathematical expressiongiven tasks than in the situation-given tasks. Furthermore, except for Task 1 (driving task), the percent of the problems posed with medium-level difficulty is higher than that of the problems with lowlevel difficulty for the other tasks. This result shows that teachers intended to pose problems at the low and medium difficulty levels.

\section{The distribution of the flexibility levels of problems posed by mathematics teachers}

Table 3 also reports the flexibility levels of the problems posed by mathematics teachers. As Table 3 shows, the flexibility levels of the problem posed were mostly at the low and medium flexibility levels. The posed problems at the high flexibility level counted less than 4 percent for each task. Furthermore, the participants/teachers posed more problems at the low flexibility level than those at the medium flexibility level for all the tasks, except Task 3 (doorbell task). These results show that teachers tended to pose problems of less flexibility. 
Table 4. Descriptive statistics of the number, difficulty, and flexibility levels of the problems posed by teachers of different teaching experience

\begin{tabular}{|c|c|c|c|c|c|}
\hline & Teaching experience & $\begin{array}{c}\text { Number of Posed } \\
\text { Problems }\end{array}$ & $M$ & $S D$ & $S E$ \\
\hline \multirow[t]{4}{*}{ Difficulty levels of all posed problem } & 1 & 8 & 1.94 & 0.48 & 0.17 \\
\hline & 2 & 34 & 1.79 & 0.32 & 0.06 \\
\hline & 3 & 25 & 1.60 & 0.30 & 0.06 \\
\hline & 4 & 14 & 1.73 & 0.43 & 0.12 \\
\hline \multirow[t]{4}{*}{ Flexibility levels of all posed problems } & 1 & 8 & 1.62 & 0.37 & 0.13 \\
\hline & 2 & 34 & 1.46 & 0.28 & 0.05 \\
\hline & 3 & 25 & 1.29 & 0.22 & 0.04 \\
\hline & 4 & 14 & 1.46 & 0.32 & 0.09 \\
\hline \multirow{4}{*}{$\begin{array}{l}\text { Difficulty levels of problems posed in } \\
\text { situation-given tasks }\end{array}$} & 1 & 8 & 2.00 & 0.53 & 0.19 \\
\hline & 2 & 34 & 1.75 & 0.49 & 0.08 \\
\hline & 3 & 25 & 1.42 & 0.43 & 0.09 \\
\hline & 4 & 14 & 1.68 & 0.54 & 0.14 \\
\hline \multirow{4}{*}{$\begin{array}{l}\text { Flexibility levels of problems posed in } \\
\text { situation-given tasks }\end{array}$} & 1 & 8 & 1.88 & 0.35 & 0.13 \\
\hline & 2 & 34 & 1.63 & 0.39 & 0.07 \\
\hline & 3 & 25 & 1.36 & 0.34 & 0.07 \\
\hline & 4 & 14 & 1.61 & 0.49 & 0.13 \\
\hline \multirow{4}{*}{$\begin{array}{l}\text { Difficulty levels of problems posed in } \\
\text { mathematical expression-given tasks }\end{array}$} & 1 & 8 & 1.87 & 0.53 & 0.19 \\
\hline & 2 & 34 & 1.83 & 0.42 & 0.07 \\
\hline & 3 & 25 & 1.78 & 0.44 & 0.09 \\
\hline & 4 & 14 & 1.79 & 0.51 & 0.14 \\
\hline \multirow{4}{*}{$\begin{array}{l}\text { Flexibility levels of problems posed in } \\
\text { mathematical expression-given tasks }\end{array}$} & 1 & 8 & 1.37 & 0.40 & 0.14 \\
\hline & 2 & 34 & 1.29 & 0.34 & 0.06 \\
\hline & 3 & 25 & 1.22 & 0.30 & 0.06 \\
\hline & 4 & 14 & 1.32 & 0.42 & 0.11 \\
\hline
\end{tabular}

Note. $1=$ Teachers with 1-5 years of teaching experience. $2=$ Teachers with $6-15$ years of teaching experience. $3=$ Teachers with 16-25 years of teaching experience. 4 = Teachers with above 25 years of teaching experience.

\section{The Relationship Between Mathematics Teachers' Problem Posing and Teaching Experience}

To examine the relationship between teaching experience and teachers' problem posing, we first conducted a descriptive analysis. Table 4 reports the mean scores of the difficulty levels and flexibility levels of the problem posed by four groups of teachers with varied years of teaching experience, i.e., Group $1=1-5$ years, Group $2=6-15$ years, Group $3=16-25$ years, and Group $4=25+$ years. As shown in Table 4 , teachers with 1-5 years of teaching experience scored the highest mean in all categories including flexibility levels for all posed problems $(M=1.62, S D=0.37)$, difficulty levels of all posed problem $(M=1.94, S D=0.48)$,difficulty levels of problems posed on situation-given tasks $(M=2.00, S D=$ $0.53)$, flexibility levels of problems posed on situationgiven tasks $(M=1.88, S D=0.35)$, flexibility levels of problems posed on mathematical expression-given tasks $(M=1.37, S D=0.40)$, and difficulty levels of problems posed in mathematical expression-given tasks $(M=1.87$, $S D=0.53)$.

A series of one-way analyses of variance (ANOVA) was conducted to examine the relationship between characteristics of the problem posed and teaching experience (Table 5). No significant difference was found in the number of problems proposed among the teachers of different teaching experiences. The overall ANOVA analyses show that there is a significant difference in the flexibility levels of the posed problems across the teachers of different experiences $\left(F_{(3,77)}=3.52, p=0.019<\right.$ $0.05)$. No significant difference was found in the difficulty levels of the posed problems among the teachers of different experiences $\left(F_{(3,77)}=2.36, p=0.078>\right.$ 0.05). One-way ANOVA was further conducted to examine the relationship of teaching experience with the difficulty and flexibility levels of the pose problems on the situation-given tasks and the mathematical expression-given tasks (Table 5). No significant differences was found for the flexibility levels $\left(F_{(3,77)}=\right.$ $0.48, p=0.698>0.05)$ and the difficulty levels $\left(F_{(3,77)}=\right.$ $0.12, p=0.947>0.05)$ for the mathematical expressiongiven tasks. However, significant differences were found for both the flexibility levels $\left(F_{(3,77)}=4.33, \quad p=0.07>0.05\right)$ and the difficulty levels $\left(F_{(3,77)}=3.76, \quad p=0.14>0.05\right)$ in the situation-given tasks.

Post-hoc analyses (LSD) were conducted to examine the influence of teaching experience on flexibility and the difficulty of the problem posed. The results show that among the four groups the teachers with 16-25 years of teaching experience scored the lowest on the flexibility levels for all problem posed $(M=1.29, S D=0.22)$, the flexibility levels of problems posed in situation-given tasks $(M=1.36, S D=0.34)$, and the difficulty levels of problems posed in situation-given tasks $(M=1.88, S D$ $=0.44)$. Their averaged score was significantly lower than those with 1-5 years of teaching experience on the 
Table 5. ANOVA results for the total numbers of problems, numbers of appropriate problems, difficulty and flexibility of the problem posed by teachers of different teaching experiences

\begin{tabular}{|c|c|c|c|c|c|c|c|c|c|c|c|}
\hline & \multicolumn{2}{|c|}{$1-5$} & \multicolumn{2}{|c|}{$6-15$} & \multicolumn{2}{|c|}{$16-25$} & \multicolumn{2}{|c|}{ above 25} & \multirow{2}{*}{$\mathrm{F}(3,77)$} & \multirow{2}{*}{ P } & \multirow{2}{*}{$\eta 2$} \\
\hline & $\mathrm{M}$ & SD & $\mathrm{M}$ & SD & $\mathrm{M}$ & SD & $\mathrm{M}$ & SD & & & \\
\hline Total numbers of problem & 12.01 & 4.08 & 9.39 & 2.90 & 8.54 & 3.06 & 9.43 & 3.65 & 2.38 & .077 & 0.08 \\
\hline Numbers of appropriate problems posed & 11.46 & 3.78 & 9.12 & 2.84 & 8.20 & 3.06 & 9.21 & 3.49 & 2.23 & .092 & 0.08 \\
\hline Difficulty levels of all problem posed & 1.94 & 0.48 & 1.79 & 0.32 & 1.6 & 0.3 & 1.73 & 0.43 & 2.36 & .078 & 0.09 \\
\hline Flexibility levels of all problems posed & 1.62 & 0.37 & 1.46 & 0.28 & 1.29 & 0.22 & 1.46 & 0.32 & 3.52 & $.019^{*}$ & 0.13 \\
\hline Numbers of pr & 7.50 & 2.07 & 5.46 & 2.30 & 4.88 & 2.40 & 5.43 & 2.21 & 2.64 & .056 & 0.09 \\
\hline $\begin{array}{l}\text { Numbers of appropriate problems posed in } \\
\text { situation-given tasks }\end{array}$ & 7.38 & 2.07 & 5.46 & 2.30 & 4.80 & 2.42 & 5.43 & 2.21 & 2.53 & .063 & 0.09 \\
\hline $\begin{array}{l}\text { Difficulty levels of problems posed in situation- } \\
\text { given tasks }\end{array}$ & 2 & 0.53 & 1.75 & 0.49 & 1.42 & 0.43 & 1.68 & 0.54 & 3.76 & $.014^{*}$ & 0.13 \\
\hline $\begin{array}{l}\text { Flexibility levels of problems posed in situation- } \\
\text { given tasks }\end{array}$ & 1.88 & 0.35 & 1.63 & 0.39 & 1.36 & 0.34 & 1.61 & 0.49 & 4.33 & $.007^{*}$ & 0.15 \\
\hline $\begin{array}{l}\text { Numbers of problems posed in mathematical } \\
\text { expression-given tasks }\end{array}$ & 4.51 & 2.33 & 3.94 & 1.15 & 3.66 & 1.34 & 4.00 & 1.71 & .73 & .537 & 0.03 \\
\hline $\begin{array}{l}\text { Numbers of appropriate problems posed in } \\
\text { mathematical expression-given tasks }\end{array}$ & 4.09 & 1.97 & 3.67 & 1.15 & 3.40 & 1.34 & 3.79 & 1.53 & .60 & .620 & 0.02 \\
\hline $\begin{array}{l}\text { Difficulty levels of problems posed in mathematical } \\
\text { expression-given tasks }\end{array}$ & 1.87 & 0.53 & 1.83 & 0.42 & 1.78 & 0.44 & 1.79 & 0.51 & .12 & .947 & 0.00 \\
\hline $\begin{array}{l}\text { Flexibility levels of problems posed in mathematical } \\
\text { expression-given tasks }\end{array}$ & 1.37 & 0.4 & 1.29 & 0.34 & 1.22 & 0.3 & 1.32 & 0.42 & .48 & .698 & 0.02 \\
\hline
\end{tabular}

Note. ${ }^{*} p<.05$

flexibility levels for all problem posed $(M=1.62, S D=$ $0.37, p=.005)$, the flexibility levels of problems posed in situation-given tasks $(M=1.88, S D=0.35, p=.002)$, and the difficulty levels of problems posed in situation-given tasks $(M=1.87, S D=0.53, p=.004)$.

\section{DISCUSSION}

This study aimed to describe characteristics of problem posing and examine its relationship with the teaching experience of elementary school mathematics teachers from ethnic minority areas in the Southwest of China. The results contribute to research on mathematical problem posing and teacher training in mathematics education. While the previous studies focused primarily on teachers from the more developed regions of China, this study paid attention to the problem posing of an under-represented group of teachers in Southwest China. Specifically, our results revealed unique characteristics and a reverse relationship between teaching experience and teachers' mathematical problem posing. Understanding characteristics of problem posing and its relationship with teaching experience has significant implications on mathematics classroom teaching and learning, mathematics education, and the professional development of mathematics teachers in the region.

\section{Characteristics of Problem Posing of Elementary School Mathematics Teachers from the Ethnic Minority Areas in Southwest China}

Our findings show that elementary school mathematics teachers from the ethnic minority areas in the Southwest of China were able to pose numerous interesting and appropriate mathematical problems on different tasks. This finding supplements Li et al.'s (2019) observation that teachers can pose interesting problems. It suggests that the elementary school mathematics teachers in the minority areas in China were as capable of posing mathematical problems as their colleagues in the more developed areas in China (Li et al., 2019).

However, the present study did not find shortcomings in conceptual understanding of certain mathematical knowledge in problem posing as reported in the previous studies (e.g., De Corte \& Verschaffel, 1996; Tichá \& Hošpesová, 2013; Toluk-Uşar, 2009; Xie \& Masingila, 2017). A possible explanation of this conflicting result could be attributed to cultural differences as our participants were Chinese teachers who possess profound knowledge (Ma, 1999) even if they came from the minority ethnic group in Southwest China. This finding points to the complexity in the study of mathematics education and highlights the importance of cultural differences in mathematics education research. Nevertheless, this does not mean that the teachers from the ethnic minority area in Southwest China are necessarily good mathematical problem posers. On the contrary, our results revealed a few quality issues of problem posing among this group of teachers, as discussed below.

\section{Problem posing in situation-given tasks and mathematical expression-given tasks}

Our results show that elementary school teachers from ethnic minority areas performed better in situationgiven tasks than mathematical expression-given tasks. This finding is consistent with Li et al's result (2019) that the teachers from the more developed areas in China 
also performed better in situation-given tasks than mathematical expression-given tasks. According to Goldin (1998), there are two different external representation systems, verbal language (i.e., the verbal representation system) determined by the use of everyday language, and symbolic representation. The latter includes specific terminology from academic mathematical language and algebraic symbolism, including letters and operational and relational symbols used in arithmetic. Earlier studies suggested it is easier for students to translate the verbal presentation to the symbolic representation system (Cañadas et al., 2018) than vice versa. This is because it is more challenging for students to grasp the meaning of the symbols in the mathematical expression. In the current study, to pose problems in the mathematical expression-given tasks, it requires the teachers to first grasp the meaning of both algebraic expressions (Faction and Equation), and then translate the task to a verbal expression. The teachers may have difficulties in giving meaning to algebraic symbolism. In contrast, the situation-given tasks require no translation between the verbal and symbolic systems. Therefore, like other colleagues, our participants performed better in the situation-given tasks than in the mathematical expression-given tasks.

\section{Quality of the problem posed by the participating mathematics teachers}

Although our results show that the teachers from the ethnic minority areas were capable of posing interesting mathematical problems, few of these problems were at high levels of difficulty and flexibility (Table 3). This result is similar to $\mathrm{Li}$ et al.'s (2019) finding that the problems posed by the teachers were mostly at the low and medium levels. However, Li et al. (2019) also found that teachers were capable of posing problems at high flexibility levels for the mathematical expression-given tasks. Both problems with low-level flexibility and closeended problems were more related to low-quality problems. In a follow-up study on the challenges of implementing problem posing in teaching, Li et al. (2020) reported that more than half of the teachers $(61 \%)$ cited that low-quality problem posing by students as an important challenge for teachers. Similarly, low-quality problems posed by teachers may also result in a problematical situation. As English (2020) pointed out that low-quality problems "display minimum learning goals, low cognitive demand, and few or no challenges, and are thus unlikely to facilitate effective teaching through problem posing" (p. 2). Therefore, our results call for more attention to improving the quality of problem-posing among the teachers from the ethnic minority areas.

\section{Cultural features of the problem posed by mathematics} teachers from ethnic minority areas

Our results indicate that teachers from ethnic minority areas in China were capable of posing mathematical problems in different situations. However, to our surprise, these teachers did not incorporate any feature and background of the ethnic minority posing the mathematics problems. There are various forms of mathematics-underlying cultural practices among the ethnic minorities in the Southwest of China, evident in their architecture, dress, drawings, counting units, chronometers, methods of calendar calculation, and religious beliefs (Peng \& Song, 2014). However, none of the posed problems was related to the local culture of the ethnic minority areas. Similarly, Zhou et al.'s (2018) reported that teachers in the ethnic minority areas lacked enough understanding of the cultural aspect of mathematics, especially in understanding the various forms of mathematics-underlying cultural practices of the ethnic minorities. These results present the need to enhance teachers' cultural sensitivity in mathematics. As Peng and Song (2014) suggested, addressing this need will better prepare teachers to pose mathematical problems in a culturally sensitive way, which, in turn, will help students grow and develop in an environment that is keen on their native cultural practices.

\section{Relationship Between Mathematics Teachers' Problem Posing and Teaching Experience}

Our results show that teacher problem posing is related to teaching experience. Most noticeably, teachers with 1-5 years of experience posed more mathematical problems at high flexibility levels than those with 6-15, 16-25, and above 25 years of teaching experience, plus with a significant difference with those of 16-25 experience. Our results also show that the problems posed by teachers varied in the flexibility and difficulty levels for the situation-given tasks. Again, the teachers with 1-5 years of experience posed more problems at high flexibility and difficulty levels for the situationgiven tasks than the teachers with 6-15, 16-25, and above 25 years of experience, and have the significant difference with teachers of 16-25 years experience. Educational research often views teaching experience as an indicator of a teacher's human capital - "an individual's cumulative abilities, knowledge, and skill developed through formal and informal education and experience" (Pil \& Leana, 2009, p. 1103). This view assumed "the more that teachers have seniority and experience, the more they know optimal ways to apply their knowledge; they have richer examples and better explanations" (Klein \& Leikin, 2020, p. 362). However, our results show that teaching experience might not necessarily function as a human capital variable for teachers in mathematical problem posing. 
One plausible explanation for this result is that problem posing is an activity that is not frequently conducted by mathematics teachers, and that most teachers have very limited experience of posing their mathematical problems. According to Klinshtern et al.'s (2015) study conducted in Israel, there were about 50\% of the participants engaged in problem posing occasionally. Similarly, the Cai et al.' study (2019) reported that the vast majority of teachers possessed very little experience with problem posing before the workshop. Therefore, having more years of teaching experience does not necessarily mean that teachers accumulated more experiences, or human capital, in problem posing. This proposition is supported by Schmitt's (2013) observation that teachers with over 30 years of experience had a negative association with students' achievement because these teachers were unable to keep up with instructional skills in education.

Another possible reason for the results relates to the contextual factor of mathematics education in China. It was not until recently that the cultivation of students' ability to pose mathematical problems was clearly stated in the Standards of Mathematics Curriculum at all levels in China (Li et al., 2020). With the Standard in place, an ever-increasing emphasis has been put on mathematical problem posing. This was reflected in the continuous updates and changes for the teacher preparation programs to meet the goals. Therefore, beginning teachers would have more exposure to the newly-added concepts such as problem posing, and be more familiar with the activities of problem posing, as compared to their colleagues who graduated decades ago. As Cai et al. (2020) suggested, a problem-posing workshop could greatly help elementary schoolteachers develop competency and confidence in problem posing, even if they had little experience in posing problems previously. We hope the workshop-based interventions could also benefit the mathematics schoolteachers in the ethnic minority area in Southwest China.

But why do teachers with 1-5 years of teaching experience pose more mathematical problems with highlevel flexibility? Our explanation is that teachers with 15 years of experience are at the beginning stage of their careers. They are more prone to independent thinking and less likely to be bound by textbooks. In contrast, more experienced teachers relied more heavily on the teaching materials and textbooks which limited their potential in divergent thinking in problem posing. As indicated in Klein and Leikin (2020), experienced teachers could instinctively ask students an open question that is related to a problem in the textbook. But for the problems with more details and more complex, they need preparation in advance. In this sense, more teaching experience is not necessarily be human capital.

Our results show teachers with 1-5 years of teaching experience posed more mathematical problems with high-level difficulty and flexibility in situation-given tasks. This is because problem posing in mathematical expression-given tasks often requires a translation between symbolic system and verbal system, while situation-given tasks usually include more verbal, sometimes informal, information and only require translation within the verbal system. It is easier for the beginning teachers who are less limited to the formal version of various rules and procedures frequently presented in mathematics textbooks to pose more problems (Popovic \& Lederman, 2015). This result may remind us that when teachers with 1-5 years of teaching experience are offered the opportunity to learn to pose mathematical problems, mathematical expression-given tasks should be given priority over situation-given tasks.

\section{Limitations}

This study generated empirical evidence on problem posing among elementary school teachers from the ethnic minority areas in Southwest China. However, our results need to be used with caution due to their limitations. First, the study relied on a relatively small sample size from a limited number of schools in the two counties in the region. Future studies with a larger sample and systemic sampling methods would help enhance the validity of the results and the generalizability of the findings to other groups and geographic locations. Second, our data source was limited to participants' written responses to a task-based questionnaire. Additional measures, e.g., think-aloud protocol and teacher reflections could be used to examine teachers' thinking process during problem posing and how teaching experiences influence problem posing in the classroom setting. Third, our study examined the relationship between teachers' mathematical problem posing and teaching experience. More demographic and contextual factors such as teacher education background, prior experiences in problem posing, and school type and size could be considered in the future study either as covariates or separate independent variables. A multivariate approach would help reveal the complexity of problemposing in mathematics teaching and education and thus enrich research on mathematics education.

\section{CONCLUSION}

The current study described characteristics of problem posing and revealed an inverse relationship between problem posing and teaching experience among elementary school mathematics teachers from ethnic minority areas in the Southwest of China. The results could help develop teacher training programs in mathematical problem posing and classroom teaching. Our results suggest that teacher professional development workshops could go beyond whether teachers can pose mathematical problems, and aim at developing teachers' ability to pose problems of quality 
measured by a high level of flexibility and difficulty. Also, the workshop could focus on teachers' ability to pose quality problems for mathematical expressiongiven tasks and to skillfully translate between the conceptual and verbal systems for mathematical thinking. Furthermore, teachers should be encouraged to pose problems based on the local cultural practice, e.g., using cultural or social artifacts and relating mathematical problems to daily-life experiences of the local communities. Finally, professional development programs could address the varied needs in problem posing training of the teachers at different stages of their professional career. Additional resources and opportunities could be considered to help the teachers who graduated years ago stay abreast with the updated curricular standards, materials, and technologies and enhance their competency in mathematical problem posing.

Author contributions: All authors have sufficiently contributed to the study, and agreed with the results and conclusions.

Funding: The authors gratefully acknowledge the grant from the National Social Science Foundation in China (17XMZ032) and Chongqing Association of Higher Education (No. CQGJ19A02) which supports the current study.

Acknowledgements: Thanks to the anonymous reviewers for the valuable comments and suggestions which led to substantial improvement of the manuscript. The authors also appreciate professor Changjun Zhou for assistance in the data collection.

Declaration of interest: No conflict of interest is declared by authors.

\section{REFERENCES}

Balka, D. (1974). Creative ability in mathematics. The Arithmetic Teacher, 21(7), 633-636. http://www.jstor.org/stable/41188634

Baumanns, L., \& Rott, B. (2020). Rethinking problemposing situations: A review. Investigations in Mathematics Learning. https://doi.org/10.1080/ 19477503.2020.1841501

Baumanns, L., \& Rott, B. (2021): Developing a framework for characterising problem-posing activities: A review. Research in Mathematics Education. https:// doi.org/10.1080/14794802.2021.1897036

Brown, S. (2001). Reconstructing school mathematics: Problems with problems and the real world. Peter Lang.

Cai, J., \& Hwang, S. (2002). Generalized and generative thinking in the US and Chinese students' mathematical problem solving and problem posing. The Journal of Mathematical Behavior, 21(4), 401-421. https://doi.org/10.1016/S0732-3123(02) 00142-6

Cai, J., \& Hwang, S. (2020). Learning to teach through mathematical problem posing: Theoretical considerations, methodology, and directions for future research. International Journal of Educational
Research, 102, 101391. https://doi.org/10.1016/ j.ijer.2019.01.001

Cai, J., \& Hwang, S. (2021). Teachers as redesigners of curriculum to teach mathematics through problem posing: Conceptualization and initial findings of a problem-posing project. ZDM Mathematics Education, 53, 1403-1416. https://doi.org/10.1007/ s11858-021-01252-3

Cai, J., \& Leikin, R. (2020). Affect in mathematical problem posing: Conceptualization, advances, and future directions for research. Educational Studies in Mathematics, 105(3), 287-301. https://doi.org/ 10.1007/s10649-020-10008-x

Cai, J., \& Mamlok-Naaman, R. (2020). Posing researchable questions in mathematics and science education: Purposefully questioning the questions for investigation. International Journal of Science and Mathematics Education, 18, 1-7. https://doi.org/ 10.1007/s10763-020-10079-5

Cai, J., Chen, T., Li, X., Xu, R., Zhang S., Hu, Y., Zhang, L., \& Song, N. (2020). Exploring the impact of a problem-posing workshop on elementary school mathematics teachers' problem posing and lesson design. International Journal of Educational Research, 102, 101404. https://doi.org/10.1016/j.ijer.2019. 02.004

Cañadas, M. C., Molina, M., \& Figueiras, A. R. (2018). Meanings given to algebraic symbolism in problem-posing. Educational Studies in Mathematics, 98, 19-37. https://doi.org/10.1007/s10649-0179797-9

Crespo, S. (2003). Learning to pose mathematical problems: Exploring changes in preservice teachers' practices. Educational Studies in Mathematics, 52, 243-270. https:// doi.org/10.1023/ A:1024364304664

De Corte, E., \& Verschaffel, L. (1996). An empirical test of the impact of primitive intuitive models of operations on solving word problems with a multiplicative structure. Learning and Instruction, 6, 219-243. https:// doi.org/10.1016/0959-4752(96)00 004-7

Einstein, A., \& Infeld, L. (1938). The evolution of physics. Simon and Schuster.

English, L. D. (2020). Teaching and learning through mathematical problem posing: Commentary. International Journal of Educational Research, 102, 101451. https:/ / doi.org/10.1016/j.ijer.2019.06.014

Goldin, G. A. (1998). Representational systems, learning, and problem solving in mathematics. Journal of Mathematical Behavior, 17(2), 137-165. https:/ / doi.org/10.1016/S0364-0213(99)80056-1

Graham, B. (1988). Mathematical education and Aboriginal children. Educational Studies in 
Mathematics, 19, 119-135. https:// doi.org/10.1007/ BF00751228

Guo, M., Leung, F. K. S., \& Hu, X. (2020). Affective determinants of mathematical problem posing: the case of Chinese Miao students. Educational Studies in Mathematics, 105, 367-387. https:/ / doi.org/10.1007/s10649-020-09972-1

Klein, S., \& Leikin, R. (2020). Opening mathematical problems for posing open mathematical tasks: what do teachers do and feel?. Educational Studies in Mathematics, 105, 349-365. https://doi.org/10.1007 / s10649-020-09983-y

Klinshtern, M., Koichu, B., \& Berman, A. (2015). Problem posing: From research to effective practice. In F. M. Singer, N. Ellerton, \& J. Cai (Eds.), What do high school teachers mean by saying "I pose my own problems"? (pp. 449-467). Lawrence Erlbaum Associates.

Leavy, A. M., \& Hourigan, M. (2020). Posing mathematically worthwhile problems: Developing the problem-posing skills of prospective teachers. Journal of Mathematics Teacher Education, 23(4), 341361. https: / / doi.org/10.1007/s10857-018-09425-W

Leavy, A., \& Hourigan, M. (2021). Balancing competing demands: Enhancing the mathematical problem posing skills of prospective teachers through a mathematical letter-writing initiative. Journal of Mathematics Teacher Education. https:/ / doi.org/10.1007/s10857-021-09490-8

Lee, S. Y. (2020). Research status of mathematical problem posing in mathematics education journals. International Journal of Science and Mathematics Education, 19, 1677-1693. https://doi.org/10.1007/ s10763-020-10128-Z

Li, X., Song, N., Chen, T., \& Cai, J. (2019). The performance of elementary mathematics teachers' problem posing. Journal of Mathematics Education, 28(2), 5-10. (In Chinese).

Li, X., Song, N., Hwang, S., \& Cai, J. (2020). Learning to teach mathematics through problem posing: Teachers' beliefs and performance on problem posing. Educational Studies in Mathematics, 105(3), 325-347. https://doi.org/10.1007/s10649-02009981-0

Liljedahl, P., \& Cai, J. (2021). Empirical research on problem solving and problem posing: a look at the state of the art. ZDM Mathematics Education, 53, 723735 https:/ / doi.org/10.1007/s11858-021-01291-w

Ma, L. (1999). Knowing and teaching elementary mathematics. LEA.

Nasir, N. S., Hand, V., \& Taylor, E. V. (2008). Culture and Mathematics in School: Boundaries between "cultural" and "domain" knowledge in the mathematics classroom and beyond. Review of
Research in Education, 32, 187-240. https:/ / doi.org/ 10.3102/0091732X07308962

Nicolaou, A. A., \& Xistouri, X. (2011). Field dependence/independence cognitive style and problem posing: An investigation with sixth grade students. Educational Psychology, 31(5), 611-627. https:/ / doi.org/10.1080/01443410.2011.586126

Ogbu, J. (1995). Understanding cultural diversity and learning. In A. B., James, \& C. M. Banks (Eds.), Handbook of research on Multicultural Education. Macmillan.

Peng, A. (2021). A framework for examining the quality of teaching for mathematical understanding in ethnic minority culture context [Paper presentation]. 14 ${ }^{\text {th }}$ International Congress of Mathematics Education, Shanghai, China, July 11-19.

Peng, A., \& Song, N. (2014). The status quo development of mathematics education for ethnic minorities in China. Studies in Ethnicity and Nationalism, 14(1), 171-187. https:/ / doi.org/10.1111/sena.12083

Peng, A., Cao, L., \& Yu, B. (2020). Reciprocal Learning in Mathematical problem posing and Problem Solving: An Interactive Study between Canadian and Chinese Elementary School Students. EURASIA Journal of Mathematics, Science and Technology Education, 16(12), em1913. https:// doi.org/10.29333/ejmste/9130

Pil, F., \& Leana, C. (2009) Applying organization research to public school reform. Academy of Management Journal, 52, 1101-1124. https:/ / doi.org/10.5465/amj.2009.47084647

Popovic, G., \& Lederman, J. S. (2015). Implications of informal education experiences for mathematics teachers' ability to make connections beyond the formal classroom. School Science and Mathematics, 115(3), 129-140. https://doi.org/10.1111/ssm. 12114

Schmitt, N. (2013). The effects of teaching experience on high school education: A statistical model on factors of educational achievement in math and science. American Journal of Agricultural Economics, 95(2), 531-535. https://doi.org/10.1093/ajae/ aas 101

Schoenfeld, A. (1989). Explorations of students' mathematical beliefs and behavior. Journal for Research in Mathematics Education, 20, 338-355. https:/ / doi.org/10.2307/749440

Silver, E. A. (1994). On mathematical problem posing. For the Learning of Mathematics, 14(1), 19-28. https:/ / doi.org/10.5951/TCM.12.3.0129

Silver, E. A., \& Cai, J. (1996). An analysis of arithmetic problem posing by middle school students. Journal for Research in Mathematics Education, 27(5), 521-539. https:/ / doi.org/10.2307/749846 
Silver, E. A., \& Cai, J. (2005). Assessing students' mathematical problem posing. Teaching Children Mathematics, 12(3), 129-135. https://doi.org/ 10.5951/TCM.12.3.0129

Tichá, M., \& Hošpesová, A. (2013). Developing teachers' subject didactic competence through problem posing. Educational Studies in Mathematics, 83, 133143. https:/ / doi.org/10.1007/s10649-012-9455-1

Toluk-Uşar, Z. (2009). Developing pre-service teachers' understanding of fractions through problem posing. Teaching and Teacher Education, 25, 166-175. https:/ / doi.org/10.1016/j.tate.2008.08.003

Van Harpen, X. Y., \& Sriraman, B. (2013). Creativity and mathematical problem posing: An analysis of high school students' mathematical problem posing in China and the USA. Educational Studies in Mathematics, 82(2), 201-221. https://doi.org/ $10.1007 /$ s10649-012-9419-5
Venkat, H., \& Askew, M. (2018). Mediating primary mathematics: Theory, concepts, and a framework for studying practice. Educational Studies in Mathematics, 97, 71-92. https://doi.org/10.1007/ s10649-017-9776-1

Xie, S., \& Cai, J. (2020). Teachers' beliefs about mathematics, learning, teaching, students, and teachers: Perspectives from Chinese high school inservice mathematics teachers. International Journal of Science and Mathematics Education, 19, 747-769. https:/ / doi.org/10.1007/s10763-020-10074-w

Zhou, C., Mu, L., Zhao, J., \& Peng, A. (2018). A case study of primary school mathematics teaching based on ethnic minority mathematical culture background. Journal of Mathematics Education, 27(3), 85-92. (In Chinese).

\section{http://www.ejmste.com}

